\title{
The Potential Impact of Delayed Radiation Therapy on Patients with Glioblastoma
}

\author{
Ibrahim Alnaami, Juliana VanderPluym, Albert Murtha, Simon Walling, \\ Vivek Mehta, Sita Gourishankar, Ambikaipakan Senthilselvan
}

\begin{abstract}
Background: Radiation therapy (RT) is the major component of glioblastoma treatment; however, the time to initiate RT after surgical intervention varies between institutions. Our study examined the time from diagnosis to the initiation of RT and its effects on overall patient survival. Methods: We retrospectively examined 267 patients with glioblastoma who received RT as part of their therapy in two Canadian tertiary care centers. The primary goal of the study is to assess if time to RT can predict/impact survival in glioblastoma patients. Results: The following variables were associated with an increased risk of death: hazard ratio (HR) of time to RT was 0.95 [95\% confidence interval (CI), 0.91-0.99] for every extra week. HRs for the type of surgery (resection or biopsy) and type of management received (standard of care in comparison with RT regardless of chemotherapeutic agents other than concomitant and adjuvant temozolomide) were 0.50 (95\% CI, 0.37-0.66) and 0.53 (95\% CI, 0.38-0.75), respectively. HR for age was 1.02 (95\% CI, 1.01-1.03) for every extra year. Standard 60 Gy RT HR was 0.70 [95\% confidence interval (CI), 0.51-0.97] in younger patients. Conclusions: The time from diagnosis to the initiation of RT was found to be a significant prognostic factor for overall patient survival. The addition of temozolomide to the treatment protocol, age, standard RT dose in younger patients and extent of surgery are others factors associated with longer survival periods.
\end{abstract}

RÉSUMÉ: Impact potentiel de la radiothérapie différée chez les patients atteints d'un glioblastome. Contexte : La radiothérapie (RT) est l'élément le plus important du traitement du glioblastome. Cependant, le moment du début de la RT après la chirurgie varie selon les institutions. Le but de l'étude était d'examiner le temps écoulé à partir du moment où le diagnostic a été posé jusqu'au moment où la RT a été commencée et ses effets sur la survie globale des patients. Méthode : Nous avons examiné rétrospectivement 267 patients atteints de glioblastome qui ont reçu de la RT dans le cadre de leur traitement dans deux centres canadiens de soins tertiaires. Le but premier de l'étude était d'évaluer si le temps écoulé jusqu'au début de la RT pouvait prédire/influencer la survie de ces patients. Résultats : Les variables suivantes étaient associées à un risque accru de décès : le rapport de risque (RR) du temps écoulé jusqu'à la RT était de 0,95 (intervalle de confiance (IC) à 95\% : 0,91 à 0,99 ) pour chaque semaine supplémentaire. Les RR pour le type de chirurgie (résection ou biopsie) et le type de traitement reçu (traitement standard par rapport à la RT, indépendamment des antinéoplasiques autres que le témozolomide administré de façon concomitante et comme adjuvant) étaient 0,50 (IC à $95 \%: 0,37$ à 0,75 ) et 0,53 (IC à $95 \%: 0,38$ à 0,75 ) respectivement. Le RR pour l'âge était 1,02 (IC à 95\%: 1,01 à 1,03) pour chaque année additionnelle. Le RR pour la RT à dose standard (60 Gy) était 0,70 (IC à 95\% : 0,51 à 0,97) chez les patients plus jeunes. Conclusions : Le temps écoulé du diagnostic au début de la RT était un facteur de prédiction significatif de la survie globale du patient. L'ajout du témozolomide au protocole de traitement, l'âge, la dose standard de RT chez les patients plus jeunes et l'étendue de la chirurgie sont d'autres facteurs associés à une survie plus longue.

Can J Neurol Sci. 2013; 40: 790-794

Glioblastoma is the most prevalent and aggressive primary malignant brain tumor in adults. ${ }^{1}$ Since the publication of Stupp et al., the standard of care for the treatment of glioblastoma includes maximal surgical resection followed by radiation therapy (RT) and concomitant and adjuvant temozolomide treatment. Despite the use of this state-of-the-art treatment regimen, the median survival time of patients with glioblastoma is only 14.6 months. ${ }^{1}$ Well-established favorable prognostic factors include the addition of RT and novel chemotherapeutic agents such as temozolomide, the maximal extent of surgical resection, younger age, and a higher Karnofsky Performance Score $^{2-5}$. The addition of RT to treatment protocols in three randomized phase III trials demonstrated an approximate doubling of survival and has been the considered standard of care since the late 1970 's. ${ }^{6-8}$ The standard time for RT for most patients is between two and six weeks following surgery ${ }^{6,9-12}$; however, in certain resource-limited centers, it may be as long as
16 weeks. ${ }^{11}$ Clinicians always advocate "the sooner the better" in regards to initiation of RT. Given that delayed RT has been found to be associated with poorer outcomes in cancers such as breast cancer and head and neck cancer ${ }^{13}$, one may expect a similar finding in the delayed initiation of RT in patients with glioblastoma.

From the Division of Neurosurgery (IA, VM), Department of Surgery, Department of Pediatric Neurology (JV), Division of Radiation Oncology (AM), Department of Oncology, Department of Medicine (SG), Department of Public Health Sciences (AS), University of Alberta, Edmonton, Alberta; Division of Neurosurgery (SW), Department of Surgery, Dalhousie University, Halifax, Nova Scotia; Division of Neurosurgery (IA), Department of Surgery, King Khalid University, Abha, Saudi Arabia.

Received July 16, 2012. Final Revisions Submitted May 8, 2013. Correspondence to: Ibrahim Alnaami, Neurosurgery Division, 2D2.01 Mackenzie Health Sciences Centre, University of Alberta, 8440112 Street, Edmonton, Alberta, T6G 2B7, Canada. Email: ialnaami@ualberta.ca 
The present study aimed to examine the time from diagnosis to the initiation of RT and its effect on overall patient survival in two Canadian tertiary care centers.

\section{MethoDS}

\section{Study Design}

The present study was based on two Canadian cities: Edmonton and Halifax. The study conducted in Edmonton, Alberta was a retrospective cohort study based on a chart and database review of patients treated at the University of Alberta Hospital, Royal Alexandra Hospital, or Cross Cancer Institute in Edmonton. The data of patients diagnosed and treated at the Queen Elizabeth II Health Sciences Center in Halifax, Nova Scotia were prospectively collected but retrospectively analyzed. The study population comprised patients treated between 2000 and 2006 at these centers. The diagnosis of glioblastoma was confirmed using a tissue sample. The primary outcome was overall survival.

\section{Study Population}

Patients ( $>18$ years old) who received RT and a tissue diagnosis of glioblastoma (either through biopsy or resection) at one of the participating centers were included in this study, which was approved by each hospital's ethics committee or review board.

The patients were classified into two groups based on the treatment they received. The first group of patients (Group A) underwent surgery (biopsy or resection) and received RT with or without any type of chemotherapeutic agent other than concomitant and adjuvant temozolomide. The second group of patients (Group B) included those who received a standard therapy comprising RT and concomitant and adjuvant temozolomide. The rationale for combining the patients who received only RT and those who received any chemotherapeutic agent other than concomitant and adjuvant temozolomide into one group is based on the lack of evidence of any other chemotherapeutic agent providing any benefit in patient survival. Therefore, all chemotherapeutic agents other than concomitant and adjuvant temozolomide were considered as nonsuccessful, and in 2001, the Medical Research Council trial concluded that no-chemotherapy control arms remain ethical in randomized trials of high-grade astrocytoma because of the lack of prolonged survival with chemotherapeutic agents. ${ }^{14}$

\section{Statistical Analyses}

All statistical analyses were performed using STATA Data Analysis and Statistical Software (Release 10. StataCorp LP, College Station, TX, USA).

Kaplan-Meier curves were used to describe survival of the two groups and Cox's proportional hazard models were used for multivariate analysis. These methods allowed for the inclusion of censored data. The outcome of survival analysis was death after surgery, and a case was censored if a patient did not die during follow-up. The censored time was defined as the time from the date of the first computed tomography (CT) or magnetic resonance imaging (MRI) at the time of diagnosis to either the date when patient was lost to follow-up or to the date of the end of the study (March 31, 2009).
Two types of variables were analyzed: continuous and categorical. The continuous variables were age, duration of symptoms (weeks), time to surgery (days), and time to RT (days). The categorical variables were gender (male or female), presence of seizure (yes or no), type of surgery (biopsy or resection), use of temozolomide (yes or no), treatment center (Edmonton or Halifax), number of surgeries $(1,>1)$, dose of RT( standard $60 \mathrm{~Gy}$, short-course RT) and entry into the clinical trial (yes or no).

The time to surgery variable was considered as the time from the date of diagnosis, which is when the patient underwent CT or MRI, to the date of surgery. The time to RT was the time from the date of diagnosis to the date when RT was initiated.

Purposeful model building was performed following multivariate analysis, which included variables with p-values $\leq 0.2$.

\section{RESULTS}

\section{Patient and Treatment Characteristics}

Our study included 267 glioblastoma patients, 161 of which were treated in Edmonton (60\%) and $106(40 \%)$ in Halifax. Of the total number of patients, $253(98 \%)$ died and $6(2 \%)$ were censored. The age variable was slightly higher in the Edmonton group [mean age, 61 years; median age, 63 years; standard deviation (SD), 11; interquartile range (IQR), 17] than in the Halifax group (mean age, 58 years; median age, 59 years; SD, 11; IQR, 14); however, the difference was insignificant. The combined overall mean, median, SD, and IQR for age were 60 years, 61 years, 11, and 15 , respectively.

The duration of symptoms showed a mean of six weeks, median of four weeks, SD of 8 , and IQR of 6 . Although the patients in Halifax presented at a relatively later time (mean, eight weeks; median, four weeks; SD, 10; IQR, 7) compared with that in Edmonton (mean, five weeks; median, four weeks; $\mathrm{SD}, 10 ; \mathrm{IQR}, 7)$, this difference was not statistically significant (Table 1).

There was some male predominance in our study as 173 (64\%) patients were male and $94(34 \%)$ were female. This trend

Table 1: Distribution of age, symptoms duration and time to surgery by centre

\begin{tabular}{llll}
\hline covariate & Mean & Median & Standard Deviation \\
Age (years) & & & \\
Edmonton & 61 & 63 & 11 \\
Halifax & 58 & 59 & 11 \\
& & & \\
Symptoms & & & \\
Duration (weeks) & 5 & 4 & 10 \\
Edmonton & 8 & 4 & \\
Halifax & & & 10 \\
& & & 9 \\
Time to Surgery (days) & 12 & 5 & 18 \\
Edmonton & 7 & 5 & \\
Halifax & & & \\
\hline
\end{tabular}


Table 2: Median of covariates by the type of management for patients received radiotherapy

\begin{tabular}{lcc}
\hline covariate & Group A & Group B \\
Age (years) & 63 & 56 \\
$\begin{array}{l}\text { Symptoms duration } \\
\text { (weeks) }\end{array}$ & 4 & 4 \\
$\begin{array}{l}\text { Time to surgery } \\
\text { (days) }\end{array}$ & 5 & 4 \\
Time to radiation therapy (days) & 45 & 49 \\
\hline
\end{tabular}

was further represented at each center: $103(64 \%)$ males and 58 (36\%) females were treated in Edmonton, whereas $70(66 \%)$ males and $36(34 \%)$ females were treated in Halifax, where 179 $(67 \%)$ patients presented with no seizures.

In terms of the type of treatment, $218(82 \%)$ patients were in group A and 49 (18\%) were in group B. In addition, $86(32 \%)$ patients underwent biopsy and 181 (86\%) underwent glioblastoma resection. Fifty patients underwent second surgery for resection (19\%), and six of them underwent more than two surgeries.

A larger portion of patients treated in Edmonton were involved in diagnostic or therapeutic trials [68 (42\%)] compared with those treated in Halifax [19 (18\%)].

The time to surgery for centers in Halifax (mean, 7 days; median, 5 days) was shorter compared with that for centers in Edmonton (mean, 12 days; median, 7 days). However, the time to RT was shorter in Edmonton (mean, 52 days; median, 48 days) than in Halifax (mean, 68 days; median, 44 days) (Table 1). Further descriptions of the data by the type of management are illustrated in Table 2 and Table 3.

As older patients (60 or older) ${ }^{15}$ may not get aggressive treatment in day to day practice, our study included 137 patients who were 60 years or older. Ninety $(65 \%)$ patients underwent surgical resection and $47(35 \%)$ underwent biopsy only, where the younger patients (130), 96 (73\%) underwent surgical resection and 34 patients had biopsy only. There was no difference in survival between elderly patients who received standard (60 Grays) vs. those who received short course (40 Grays) in the same group.

\section{Univariate Analysis}

Univariate analysis showed no significant difference between the two centers or genders. However, the following variables showed a significant relationship with patient survival in univariate analysis: age [hazard ratio (HR), 1.02; 95\% confidence interval (CI), 1.01-1.03], involvement in trials (HR, 0.68 ; 95\% CI, 0.52-0.89), type of management [concomitant temozolomide (HR, 0.58; 95\% CI, 0.41-0.81)] (Figure 1), and type of surgery [surgical resection (HR, $0.53 ; 95 \% \mathrm{CI}, 0.40$ 0.700 ) compared with biopsy]. The time to RT (weeks) was not significant by univariate analysis (HR, $0.96,95 \% \mathrm{CI}, 0.92-1.00$ ). Dose of radiation demonstrated a direct relationship with
Table 3: Characteristics of overall patients by treatment groups *

\begin{tabular}{lcc}
\hline covariate & Group A & Group B \\
$\begin{array}{l}\text { Gender } \\
\text { Female } \\
\text { Male }\end{array}$ & $79(30)$ & \\
& $139(52)$ & $15(5)$ \\
Surgery type & & \\
$\quad$ Biopsy & & \\
Resection & $63(13)$ \\
Seizure & $155(58)$ & $18(7)$ \\
No & & $31(11)$ \\
Yes & $144(54)$ & \\
Trials & $74(28)$ & $35(13)$ \\
No & & $14(5)$ \\
Yes & $152(57)$ & \\
\hline
\end{tabular}

*N.B: Rounded percentages are indicated in parenthesis.

survival (HR 0.72, $95 \%$ CI 0.65-0.82) for each additional gray, as well as a protective effect of patients who underwent more than one surgery (HR 0.69, 95\% CI, 0.49- 0.96).

\section{Multivariate Analysis}

For multivariate analysis, eight variables with p-values $<0.2$ were included: age, duration of symptoms, presence of seizures, type of surgery, type of treatment, patient involvement in trials, time to surgery, and time to RT. The final model showed that four variables significantly contributed to patient survival: age (HR, $1.02 ; 95 \% \mathrm{CI}, 1.01-1.03)$, type of surgery [the resection group (HR, 0.50; 95\% CI, 0.37-0.66) compared with the biopsy group], type of management [group A ( $\mathrm{HR}=0.53$; 95\% CI, 0.38-0.75) compared with group B], and time to RT (HR, 0.95; 95\%CI, 0.91-0.99) (Figure 2), i.e., in any given glioblastoma patient who survive between 12-14 months, each week of delay in RT results in 18-21 days reduction in survival.

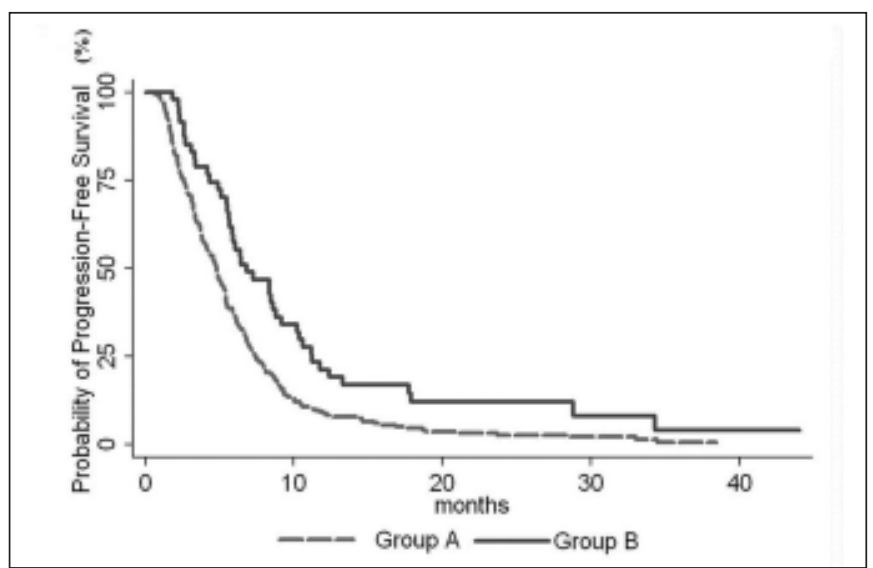

Figure 1: Kaplan-Meier estimates of survival by the type of management. 


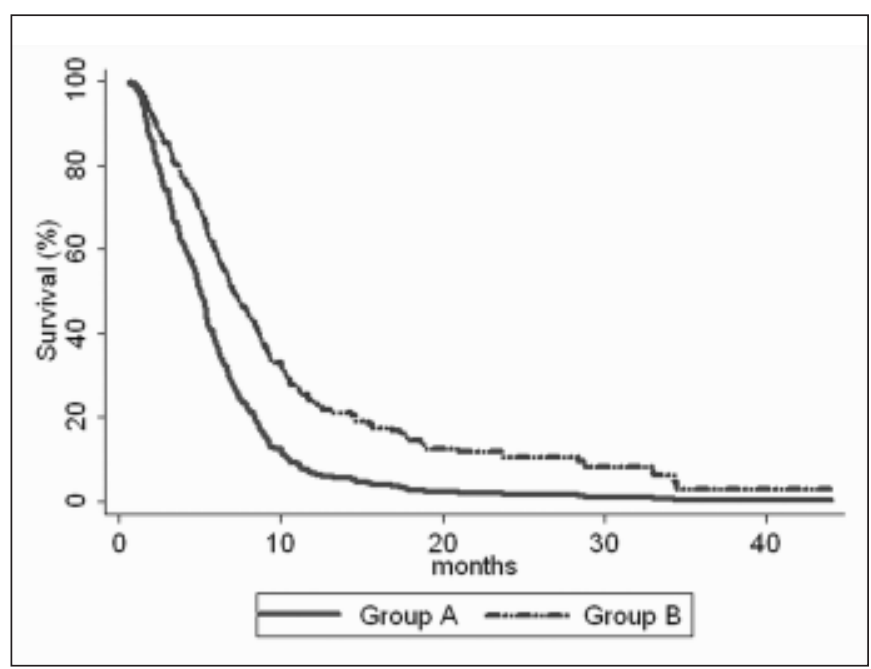

Figure 2: Adjusted estimates of survival from Cox's proportional hazards regression of the type of management group.

\section{Discussion}

Our retrospective cohort study found that the time from diagnosis of glioblastoma by neuroimaging to the initiation of RT was a significant prognostic factor for overall survival and that there was an inverse relationship between the time and survival. Some conflicting studies have examined the optimal timing of RT in the treatment of glioblastoma; ${ }^{9-12,16,17}$ however, most studies analyzed patients from the pre-temozolomide era. Through retrospective analysis, Do et al studied 182 patients with grade III/IV gliomas from 1979 to $1995^{10}$ and reported that the median wait times for the initiation of RT were 26 days from biopsy and 15 days from presentation. They found on multivariate analysis that a longer time from presentation to the initiation of RT was associated with shorter survival and that the risk of death increased by $2 \%$ per day of delay. However, they did not find a statistically significant association between the time from biopsy to the initiation of RT and survival. Irwin et al retrospectively analyzed the time from surgery to the initiation of RT in 172 patients from 1993 to $2003 .{ }^{11}$ They found a mean wait time of 35 days based on multivariate analysis, which was significantly associated with survival with an $8.9 \%$ increase in the risk of death per additional week of delay in RT. These results were similar to those of Burnet et al, who developed a mathematical model for analyzing the data of patients with glioblastoma and found that delayed RT led to a negative prognosis. ${ }^{18}$

Blumenthal et al retrospectively analyzed 2,855 patients from 1974 to $2003^{9}$ and reported that the time from surgery to the start of RT should be a maximum of six weeks as per the Radiation Therapy Oncology Group protocol. In their analysis, the median survival time in a group with a delay of more than four weeks in the initiation of RT was 12.5 months compared with 9.2 months in a group with a delay of less than two weeks. The authors noted that patients receiving RT within two weeks of surgery were more likely to have undergone biopsy only, had a poorer response, and had worse neurologic impairment, all of which are known prognostic factors associated with poor outcomes..$^{2-5}$ In an attempt to explore these confounding factors, Blumenthal et al studied the association between the time to RT and early tumor progression as a surrogate marker of a poor prognosis; however, they found no relationship to suggest that patients with poorer prognoses were clumped in the early RT group. Using the retrospective data from the Surveillance, Epidemiology, and End Result Medicare database, Lai et al analyzed 1,375 patients from 1991 to $2002^{12}$ by focusing on $>65$-year-old patients, a group known to have a poorer prognosis. ${ }^{19}$ Similar to the studies by Blumenthal and Irwin et al., the wait time was measured as the time from surgery to the initiation of RT and the outcome was overall survival. The median time to start RT was 15 days. The type of surgery was used as a stratification factor in further analyses as the authors found that RT was initiated six days earlier in patients who underwent biopsy than in those who underwent debulking surgery. Initially, univariate analysis of patients who underwent debulking surgeries with a wait time of $>22$ days showed a significant inverse relationship with survival. However, in the final Cox model, the wait time was not a significant prognostic factor. In patients who underwent biopsies only, the wait time was not a significant prognostic factor in both univariate and multivariate analyses.

The most recent study published in 2012 by Wehming et al was a single institution retrospective analysis of 153 patients with grade III/IV gliomas between 2002 and 2008. ${ }^{17}$ By univariate analysis, they showed that a time of more than 24 days between surgery and RT was associated with improved outcome for both progression-free survival and overall survival. However, multivariate analysis indicated that only the grade and use of chemotherapy remained as statistically significant positive prognostic factors for overall survival. A recent review of these heterogeneous studies has proposed that moderate postoperative wait times, no longer than six weeks, are safe and modestly beneficial. ${ }^{20}$

A unique concept of the present study was to consider the time of radiological diagnosis and clinical suspicion of glioblastoma to the time of definitive RT. This concept is more relevant to Canadian patients where access to care; including surgery and radiation therapy may be different from patients in the United States or Europe. Multiple factors may influence the period of time a patient may have to wait for radiotherapy. One may argue that each stage of the treatment / investigative paradigm may ultimately influence outcome.

It goes without saying that treatment of patients with glioblastoma is a "changing landscape". Any retrospective review that bridges a time period where the "standard treatment" has changed will have results that must be tempered. Our study population was exposed to different types of treatment, but irrespective of whether patients were in Group A and B, time to radiation therapy is an essential factor, regardless of protocol.

In concordance with a previous study, ${ }^{19}$ we found that older age is a significant prognostic factor for decreased survival. The type of surgery (biopsy or resection) showed a protective mechanism for patients who underwent resection compared with those who underwent biopsy only. The resection group in our study combined both groups of patients who underwent partial and total resection..$^{21}$ Nonetheless, the significance of this factor for predicting survival was very high. RT with concomitant temozolomide showed a significant improvement in survival as shown in the literature..$^{22,23}$ 
The gender, duration of symptoms, and time to surgery were not significant in the prediction of survival, in addition to presence of seizure. ${ }^{24}$ Some studies showed that seizure is a protective factor where patients might seek medical attention earlier, however; this remains as controversial factor. ${ }^{24,25}$ The time to surgery variable was insignificant, possibly because of the short time from the diagnosis of glioblastoma by CT or MRI to the time of surgery compared with the much longer time to the initiation of RT. As per previous studies, our data showed that short-course RT in the elderly population was insignificant in prediction of survival. ${ }^{15}$

The present study had several limitations, including the inherent limitations of its retrospective nature. The unavailability of the Karnofsky Performance Score in the patients' charts was a limiting factor, especially in comparing the results of the present study with those of other studies. Pooling of the data "Halifax prospectively collected data; that was retrospectively analysed and Edmonton retrospectively collected data" is considered another limitation of our study. Because the study was retrospective in nature, selection bias was also present in many aspects, starting from the neurosurgeon who checks the patient and decides what type of surgical intervention is warranted (biopsy or resection) to the radiation oncologist who decides if the patient requires RT as an intervention, and if so, the type of RT required, to finally the neuro-oncologist who decides whether to prescribe chemotherapeutic agents. Recall bias was also present in two variables: presence of seizure and duration of symptoms prior to presentation. Lastly, the double nature of the collected data was a limiting factor because the data from Halifax were prospectively collected, whereas those from Edmonton were retrospectively collected.

\section{Conclusions}

Our retrospective cohort study showed that a shorter time to $\mathrm{RT}$ is a potential factor in improving patient survival by offering a modifiable factor to the treatment team. As previously shown in the literature, a younger age, surgical resection, standard RT dose in younger patients, and RT with concomitant adjuvant temozolomide yield survival benefits.

\section{REFERENCES}

1. Stupp R, Dietrich PY, Ostermann Kraljevic S, et al. Promising survival for patients with newly diagnosed glioblastoma multiforme treated with concomitant radiation plus temozolomide followed by adjuvant temozolomide. J Clin Oncol. 2002;20(5):1375-82.

2. Darefsky AS, King JT, Jr., Dubrow R. Adult glioblastoma multiforme survival in the temozolomide era: a population-based analysis of Surveillance, Epidemiology, and End Results registries. Cancer. 2012;118(8):2163-72.

3. Gerstein J, Franz K, Steinbach JP, Seifert V, Rodel C, Weiss C. Radiochemotherapy with temozolomide for patients with glioblastoma. Prognostic factors and long-term outcome of unselected patients from a single institution. Strahlenther Onkol. 2011;187(11):722-8

4. Lawrence YR, Blumenthal DT, Matceyevsky D, Kanner AA, Bokstein F, Corn BW. Delayed initiation of radiotherapy for glioblastoma: how important is it to push to the front (or the back) of the line? J Neurooncol. 2011;105(1):1-7.

5. McGirt MJ, Chaichana KL, Gathinji M, et al. Independent association of extent of resection with survival in patients with malignant brain astrocytoma. J Neurosurg. 2009;110(1):156-62.
6. Keime-Guibert F, Chinot O, Taillandier L, et al. Radiotherapy for glioblastoma in the elderly. N Engl J Med. 2007;356(15): 1527-35.

7. Kristiansen K, Hagen S, Kollevold T, et al. Combined modality therapy of operated astrocytomas grade III and IV. Confirmation of the value of postoperative irradiation and lack of potentiation of bleomycin on survival time: a prospective multicenter trial of the Scandinavian Glioblastoma Study Group. Cancer. 1981;47 (4):649-52.

8. Walker MD, Alexander E, Jr., Hunt WE, et al. Evaluation of BCNU and/or radiotherapy in the treatment of anaplastic gliomas. A cooperative clinical trial. J Neurosurg. 1978;49(3):333-43.

9. Blumenthal DT, Won M, Mehta MP, et al. Short delay in initiation of radiotherapy may not affect outcome of patients with glioblastoma: a secondary analysis from the radiation therapy oncology group database. J Clin Oncol. 2009;27(5):733-9.

10. Do V, Gebski V, Barton MB. The effect of waiting for radiotherapy for grade III/IV gliomas. Radiother Oncol. 2000;57(2):131-6.

11. Irwin C, Hunn M, Purdie G, Hamilton D. Delay in radiotherapy shortens survival in patients with high grade glioma. J Neurooncol. 2007;85(3):339-43.

12. Lai R, Hershman DL, Doan T, Neugut AI. The timing of cranial radiation in elderly patients with newly diagnosed glioblastoma multiforme. Neurooncol. 2010;12(2):190-8.

13. Chen Z, King W, Pearcey R, Kerba M, Mackillop WJ. The relationship between waiting time for radiotherapy and clinical outcomes: A systematic review of the literature. Radiother Oncol. 2008;87(1):3-16.

14. Randomized trial of procarbazine, lomustine, and vincristine in the adjuvant treatment of high-grade astrocytoma: a Medical Research Council trial. J Clin Oncol. 2001;19(2):509-18.

15. Roa W, Brasher PM, Bauman G, et al. Abbreviated course of radiation therapy in older patients with glioblastoma multiforme: a prospective randomized clinical trial. J Clin Oncol. 2004;22 (9): $1583-8$

16. Lawrence YR, Mishra MV, Werner-Wasik M, et al. Improving prognosis of glioblastoma in the 21st century: who has benefited most? Cancer. 2012;118(17):4228-34.

17. Wehming FM, Wiese B, Nakamura M, Bremer M, Karstens JH, Meyer A. Malignant glioma grade 3 and 4: how relevant is timing of radiotherapy? Clin Neurol Neurosurg. 2012;114(6):617-21.

18. Burnet NG, Jena R, Jefferies SJ, Stenning SP, Kirkby NF. Mathematical modelling of survival of glioblastoma patients suggests a role for radiotherapy dose escalation and predicts poorer outcome after delay to start treatment. Clin Oncol (R Coll Radiol). 2006;18(2):93-103.

19. Burger PC, Green SB. Patient age, histologic features, and length of survival in patients with glioblastoma multiforme. Cancer. 1987; 59(9):1617-25.

20. Lawrence YR, Mishra MV, Werner-Wasik M, et al. Improving prognosis of glioblastoma in the 21st century: who has benefited most? Cancer. 2012;118(17):4228-34.

21. Hulshof MC, Koot RW, Schimmel EC, Dekker F, Bosch DA, Gonzalez Gonzalez D. Prognostic factors in glioblastoma multiforme. 10 years experience of a single institution. Strahlenther Onkol. 2001;177(6):283-90.

22. Mason WP, Maestro RD, Eisenstat D, et al. Canadian recommendations for the treatment of glioblastoma multiforme. Curr Oncol. 2007;14(3):110-17.

23. Stupp R, Mason WP, van den Bent MJ, et al. Radiotherapy plus concomitant and adjuvant temozolomide for glioblastoma. $\mathrm{N}$ Engl J Med. 2005;352(10):987-96.

24. Li J, Wang M, Won M, et al. Validation and simplification of the Radiation Therapy Oncology Group recursive partitioning analysis classification for glioblastoma. Int J Radiat Oncol Biol Phys. 2011;81(3):623-30.

25. Ozbek N, Cakir S, Gursel B, Meydan D. Prognostic significance of seizure in patients with glioblastoma multiforme. Neurol India. 2004;52(1):76-8. 